\title{
Accelerated neurodegeneration through chaperone-mediated oligomerization of tau
}

\author{
Laura J. Blair, ${ }^{1}$ Bryce A. Nordhues, ${ }^{1}$ Shannon E. Hill, ${ }^{2}$ K. Matthew Scaglione, ${ }^{3}$ \\ John C. O'Leary III,1 Sarah N. Fontaine, ${ }^{1}$ Leonid Breydo,, ${ }^{1}$ Bo Zhang, ${ }^{1}$ Pengfei Li, ${ }^{1}$ Li Wang, ${ }^{1}$ \\ Carl Cotman, ${ }^{4}$ Henry L. Paulson, ${ }^{3}$ Martin Muschol, ${ }^{2}$ Vladimir N. Uversky, ${ }^{1,5}$ Torsten Klengel, ${ }^{6}$ \\ Elisabeth B. Binder, ${ }^{6}$ Rakez Kayed,, ${ }^{7}$ Todd E. Golde, ${ }^{8}$ Nicole Berchtold, ${ }^{4}$ and Chad A. Dickey ${ }^{1}$ \\ 1Department of Molecular Medicine, Byrd Institute, and 2Department of Physics, University of South Florida, Tampa, Florida, USA. \\ ${ }^{3}$ Department of Neurology, University of Michigan, Ann Arbor, Michigan, USA. ${ }^{4}$ Institute for Memory Impairments and Neurological Disorders, \\ University of California, Irvine, California, USA. Institute for Biological Instrumentation, Russian Academy of Sciences, Moscow, Russia. \\ ${ }^{6}$ Max Planck Institute of Psychiatry, Munich, Germany. ${ }^{7}$ Department of Neurology and Department of Neuroscience and Cell Biology, \\ George P. and Cynthia Woods Mitchell Center for Neurodegenerative Diseases, The University of Texas Medical Branch, \\ Galveston, Texas, USA. ${ }^{8}$ Department of Neuroscience, University of Florida, Gainesville, Florida, USA.
}

\begin{abstract}
Aggregation of tau protein in the brain is associated with a class of neurodegenerative diseases known as tauopathies. FK506 binding protein $51 \mathrm{kDa}$ (FKBP51, encoded by FKBP5) forms a mature chaperone complex with Hsp90 that prevents tau degradation. In this study, we have shown that tau levels are reduced throughout the brains of $\mathrm{Fkpp5}^{-/-}$mice. Recombinant FKBP51 and Hsp90 synergized to block tau clearance through the proteasome, resulting in tau oligomerization. Overexpression of FKBP51 in a tau transgenic mouse model revealed that FKBP51 preserved the species of tau that have been linked to Alzheimer's disease (AD) pathogenesis, blocked amyloid formation, and decreased tangle load in the brain. Alterations in tau turnover and aggregate structure corresponded with enhanced neurotoxicity in mice. In human brains, FKBP51 levels increased relative to age and $\mathrm{AD}$, corresponding with demethylation of the regulatory regions in the FKBP5 gene. We also found that higher FKBP51 levels were associated with AD progression. Our data support a model in which age-associated increases in FKBP51 levels and its interaction with Hsp90 promote neurotoxic tau accumulation. Strategies aimed at attenuating FKBP51 levels or its interaction with Hsp90 have the potential to be therapeutically relevant for $\mathrm{AD}$ and other tauopathies.
\end{abstract}

\section{Introduction}

Neurofibrillary tangles, hallmarks of a class of neurodegenerative diseases termed tauopathies, are comprised of microtubule-associated tau protein aggregates that persist in the brain even after neuron death (1). In the most common tauopathy, Alzheimer's disease (AD), the presence of these tangles correlates with pathological development and disease progression. However, levels of neuronal death in $\mathrm{AD}$ far exceed what is expected based on the observed numbers of tau tangles (2), suggesting that a different mechanism is primarily responsible for neurodegeneration. One possible explanation for this discrepancy is that soluble tau species not visible by standard histological methods are the main cause of neurotoxicity in tauopathies (3). In fact, neurons may form tangles as a protective mechanism to sequester these more toxic soluble intermediates and convert them into a less harmful form $(4,5)$. These soluble tau species may also be able to spread from neuron to neuron in a fashion similar to that of prions, further establishing their role in perpetuating pathogenicity (6-8). Mutations in MAPT, the gene encoding tau, can also lead to the production of soluble tau conformers with pathogenic potential, leading to rarer tauopathies, such as progressive supranuclear palsy and frontal temporal dementia with parkinsonism linked to chromosome 17 (9-11). Thus, identifying the mechanisms that contribute to the production of soluble tau intermediates could be paramount for developing therapeutic approaches to treat $\mathrm{AD}$ and other tauopathies.

Conflict of interest: The authors have declared that no conflict of interest exists. Citation for this article: JClin Invest. 2013;123(10):4158-4169. doi:10.1172/JCI69003.
The variability in age of onset for $\mathrm{AD}$ and other tauopathies, even those caused by bona fide hereditary mutations, indicates that other environmental- and experience-based genetic modifiers are involved in the production of pathogenic tau species $(12,13)$. One group of age-affected genes that can also modulate tau proteostasis is the molecular chaperone family, which includes heat shock proteins (Hsps), proteins whose expression increases under conditions of stress (14). Levels of Hsps diminish with age, and roles for these proteins in neurodegenerative disease in relation to the age of onset have been proposed in several models (15). In particular, the Hsp90 system is of interest as a potential tau "toxifier" $(16,17)$.

We and others have shown that inhibiting Hsp90 can promote tau clearance, suggesting that Hsp90 is acting to protect tau in neurons for unknown reasons $(16,18,19)$. Hsp90 can even enhance tau amyloid formation in vitro, a unique function in comparison with those of other chaperones. Both of these functions of Hsp90 are in contrast to the effects of other chaperones that have been shown to enhance tau clearance and subvert its amyloidogenesis (20). Though several cochaperones of Hsp90 can influence tau stability in cells, this has not been well studied in the brain. For instance, the FK506 binding protein $51 \mathrm{kDa}$ (FKBP51, encoded by FKBP5) is known to work with Hsp90 to stabilize tau and regulate tau phosphorylation (17), but the mechanisms and pathological relevance for both of these processes are unknown. FKBP51 possesses properties that are similar to those of the cis -trans peptidyl-prolyl isomerase Pin1, which has been reported on extensively (21-23); however, much less is known about the role of FKBP51 in the brain despite its genetic association to human 
A

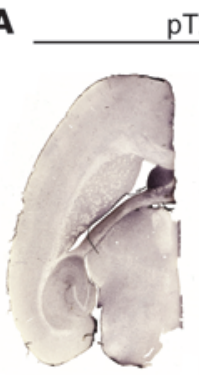

pT231

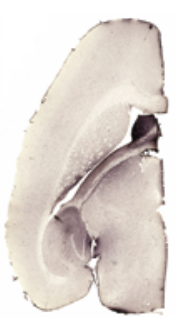

B

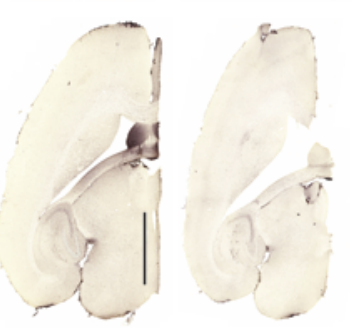

No Primary

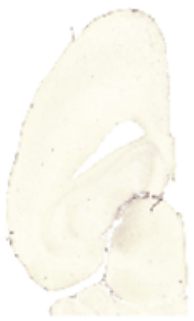

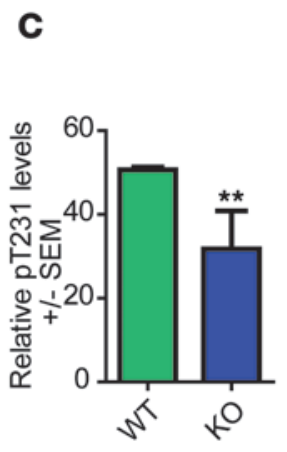

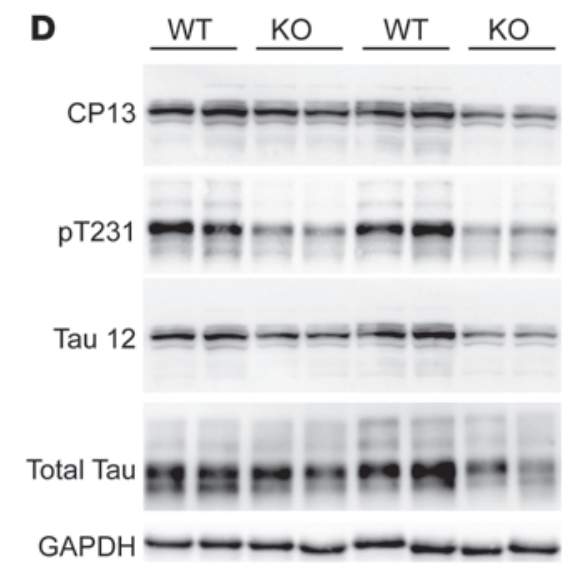

E

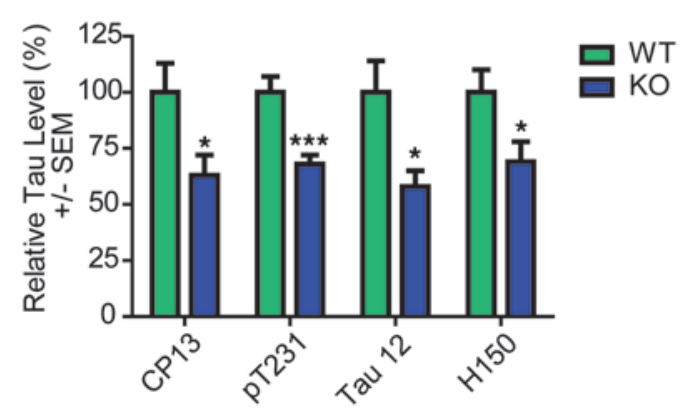

F
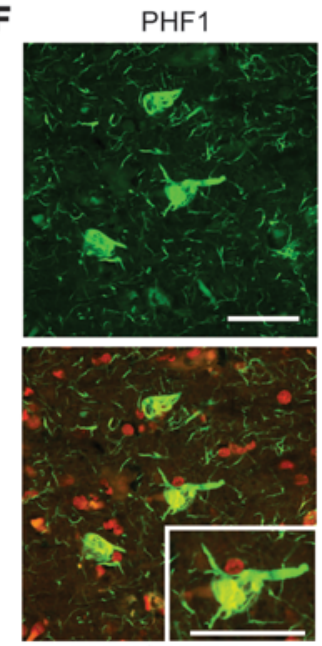

PHF1/FKBP51
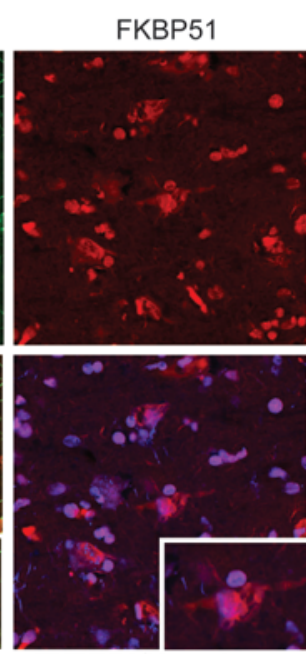

FKBP51/Neurotrace
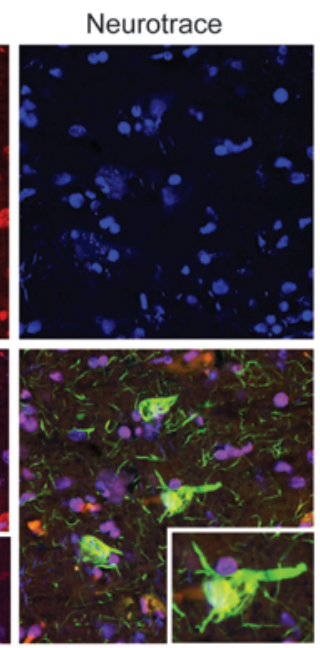

Merge

\section{Figure 1}

Tau expression is decreased in the Fkbp5/-- mice and associates with FKBP51 in the AD brain. Representative images of brain tissue from (A) wild-type and (B) Fkbp5 $5^{-1-}$ mice stained for pT231 tau and corresponding sections without primary (no primary). Scale bar: 2,000 $\mu \mathrm{m}$. (C) Quantification of pT231 stain $\left( \pm\right.$ SEM). ${ }^{* \star} P=0.0097$. (D) Representative Western blot of brain homogenates from $F k b p 5^{-/-}$mice probed for CP13 (pS202/pT205), Tau 12 (total tau), pT231 tau, H150 (total tau), and GAPDH. (E) Quantification of duplicate Western blots $\left( \pm\right.$ SEM). ${ }^{*}<<0.05$ for CP13, Tau 12 (total tau), and H150 (total tau); ${ }^{* *} P<0.001$ for pT231. (F) Fluorescent micrograph of human cortex stained with FKBP51, PHF1 (pS396/pS404), and Neurotrace (neuronal nuclei) antibodies (original magnification, $\times 60$ ). Scale bar: $50 \mu \mathrm{m}$.

psychiatric diseases (24). FKBP51 is a profolding Hsp90 cochaperone that forms a complex with Hsp90 via the tetratricopeptide (TPR) domain $(25,26)$, a region also found on other Hsp90 cochaperones, including the carboxyl terminus of Hsp70-interacting protein (CHIP; also known as STUB1) and FK506 binding protein 52 (FKBP52; also known as FKBP4) $(27,28)$. However, the TPR cochaperone with the highest affinity for Hsp90 is FKBP51 (29). Here we investigate how the FKBP51/ Hsp90 complex prevents clearance of tau, the impact it has on tau pathogenesis, and whether it plays a role in disease onset due to age-associated changes in TPR protein levels in the human brain. Our findings suggest that environmental or experiential-based epigenetic changes that accelerate upregulation of FKBP51 over time could be a major contributor to the onset and pathogenicity of tauopathies and, in particular, AD.

\section{Results}

FKBP51 depletion reduces tau levels in brain. Previous work showed that FKBP51 depletion reduced tau levels in cell models of tauopathy (17). However, it was not clear whether this was relevant in vivo. To address this issue, brain tissue from $F k b p 5$ knockout mice was collected and subjected to both immunohistochemical and biochemical analyses. Mice were generated as previously described $(30,31)$. Staining of $\mathrm{Fkbp5}^{-/-}$mice revealed a significant reduction in tau levels throughout the brain (Figure 1, A-C). Similarly, Western blot analyses of brain homogenates showed similar reductions in several tau epitopes, including a significant reduction in total tau (as assessed with the Tau 12 [amino acids 2-18] and H150 [tau $\mathrm{N}$ terminus] antibodies) and phosphorylated tau (as assessed with the CP13 [pS202] and pT231 [PHF6] tau antibodies) (Figure 1, $\mathrm{D}$ and $\mathrm{E})$. We then performed fluorescent analyses to confirm 
A

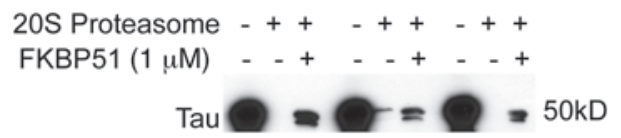

B
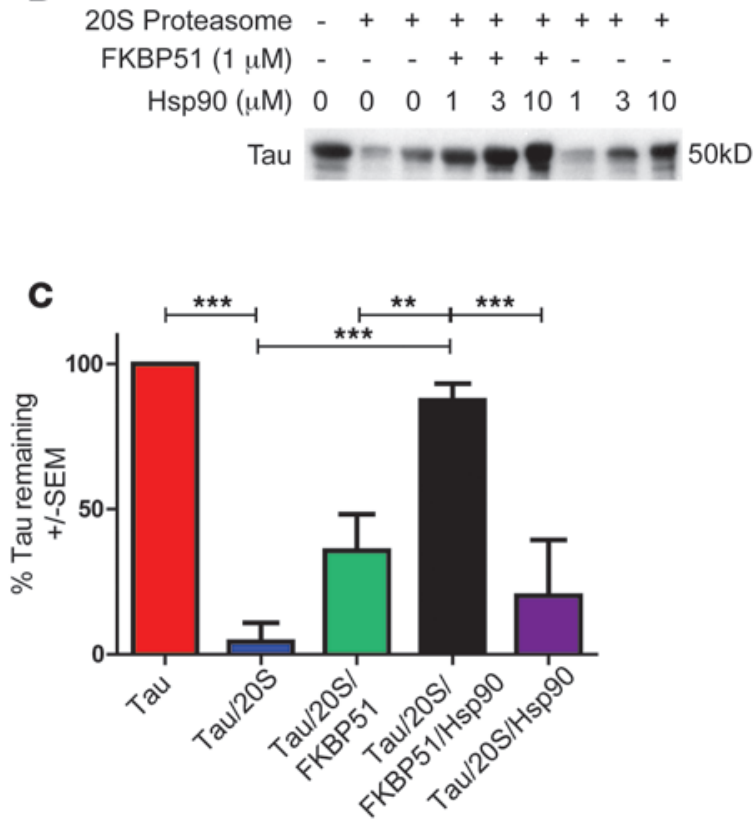

D

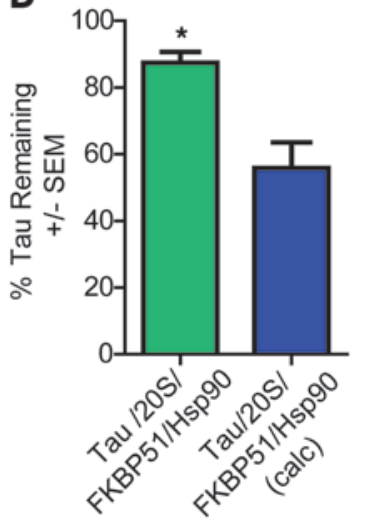

E

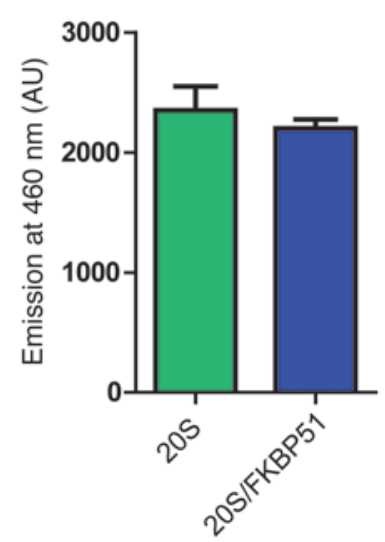

Figure 2

FKBP51 and Hsp90 synergistically prevent tau degradation by the $20 \mathrm{~S}$ proteasome. (A) Tau was incubated alone, with 20S proteasome, or with FKBP51 and $20 \mathrm{~S}$ proteasome for 5 minutes. (B) Tau was incubated alone, with $20 \mathrm{~S}$ proteasome, with FKBP51, and with Hsp90 with or without FKBP51 for 5 minutes. (C) Relative tau levels ( \pm SEM) in each sample using $1 \mu \mathrm{M}$ Hsp90. ${ }^{* *} P<0.01$, ${ }^{\star * \star} P<0.001$. (D) Tau levels ( \pm SEM) following incubation with FKBP51 and Hsp90 in the presence of the $20 \mathrm{~S}$ proteasome (green). Calculated tau levels (blue) from the addition of the results of tau preserved following incubation with $\mathrm{Hsp90}$ and with the tau preserved following incubation with FKBP51 in the presence of the 20S proteasome. ${ }^{*} P<0.05$. (E) FKBP51 was incubated with $100 \mu \mathrm{g}$ Suc-LLVY-AMC and $20 S$ proteasome for 5 minutes. OD readings $( \pm$ SEM) were taken at $460 \mathrm{~nm}$. that FKBP51 and tau were colocalized in neurons in human brain. Immunostaining with FKBP51 antibody and PHF1 (pS396/S404 tau) antibody that stains human $\mathrm{AD}$ brain well, combined with a neuronal marker, showed colocalization of neuronal tau pathology and FKBP51 in AD cortex (Figure 1F).

FKBP51 works with Hsp90 to synergistically block the 20 S proteasome from degrading tau. Previously we reported that FKBP51 coordinated with Hsp90 to promote the accumulation of nonubiquitinated tau in the presence of a proteasome inhibitor (17). This suggested that FKBP51 may be playing a role in ubiquitin-independent tau degradation via the $20 \mathrm{~S}$ proteasome, a process shown to be a major route of tau clearance $(32,33)$. The $20 \mathrm{~S}$ proteasome serves as the catalytic core for the $26 \mathrm{~S}$ proteasome (34), and $20 \mathrm{~S}$ proteasomes can be about 3 - to 4-times more abundant in cells than $26 \mathrm{~S}$ proteasomes (35). In addition, FKBP51 was previously found to slow the degradation of tau by chymotrypsin, the major protease of the $20 \mathrm{~S}$ proteasome (17). To determine unequivocally whether the FKBP51/Hsp90 complex could alter the kinetics of clearing tau by the $20 \mathrm{~S}$ proteasome, we used a reconstituted in vitro assay, as previously described (32). Indeed, tau was rapidly degraded by the $20 \mathrm{~S}$ proteasome, but this was partially blocked by FKBP51 alone (Figure 2A). When Hsp90 and FKBP51 were combined, tau degradation by $20 \mathrm{~S}$ proteasome was completely blocked (Figure 2, B and C), whereas Hsp90 alone had effects on tau similar to those of FKBP51 alone. The effects of FKBP51 and Hsp90 together were significantly greater than the additive effects of each individually, suggesting true synergy (Figure 2D). Changes in degradation were not attributed to nonspecific alteration of the $20 \mathrm{~S}$ proteasome, as the 20 S reporter sub- strate was not affected by the addition of FKBP51 (Figure 2E). Thus, FKBP51 and Hsp90 synergized to prevent 20S proteasome-mediated tau clearance, implying that this chaperone complex not only is involved in tau turnover, but possibly also suggesting a novel mechanism for regulating intracellular 20S proteasome substrates.

FKBP51 coordinates with Hsp90 to produce nonamyloid, Thioflavin Tnegative tan oligomers. The synergistic effects of Hsp90 and FKBP51 directly on tau proteasomal degradation suggested that these 2 chaperones somehow worked together to regulate tau conformation and possibly its aggregation kinetics. To get a general idea about how FKBP51 and Hsp90 regulated tau structure, circular dichroism (CD) analyses were performed with tau in combination with FKBP51, Hsp90, or both proteins together. As previously shown, tau is an intrinsically disordered protein, and its CD spectra have a minima at $200 \mathrm{~nm}$ (36). Indeed, the tau produced in our lab reproduced these results, showing a spectrum from 190 to $260 \mathrm{~nm}$, typical for a highly disordered protein such as tau (Figure 3A). The secondary structures of FKBP51 and Hsp90 were also determined by CD. Consistent with previous reports, FKBP51 and Hsp90 showed substantial ordered secondary structure $(28,37)$. The PONDR-FIT algorithm, one of the most accurate disorder predictors (38), confirmed the CD data, indicating that tau is almost completely disordered, while FKBP51 and Hsp90 are largely ordered (Supplemental Figure 1; supplemental material available online with this article; doi:10.1172/JCI69003DS1). Next, CD spectra were collected for tau in the presence of FKBP51 alone, Hsp90 alone, or both chaperones combined (Figure 3B). The dashed lines in Figure 3B show the predicted spectra of these protein mixtures if no structural change were 
A
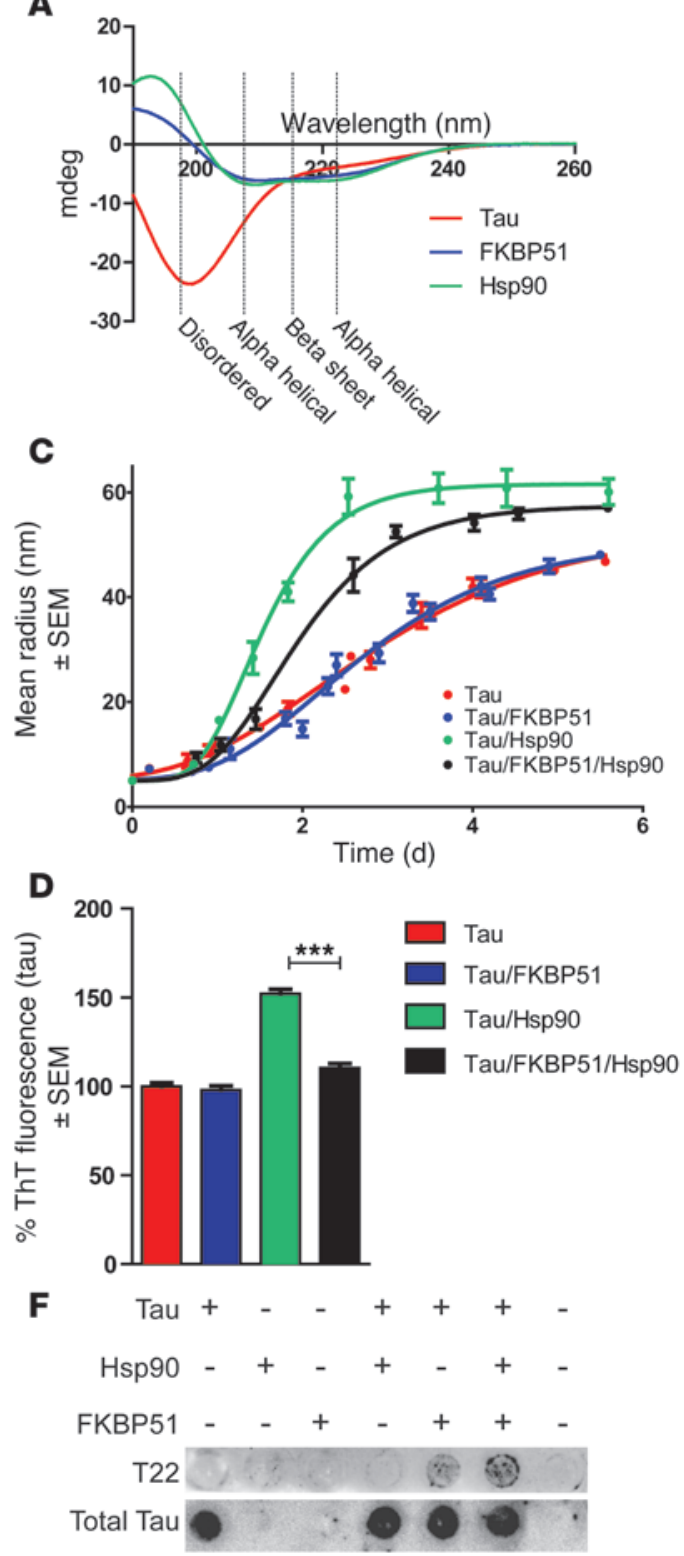

B

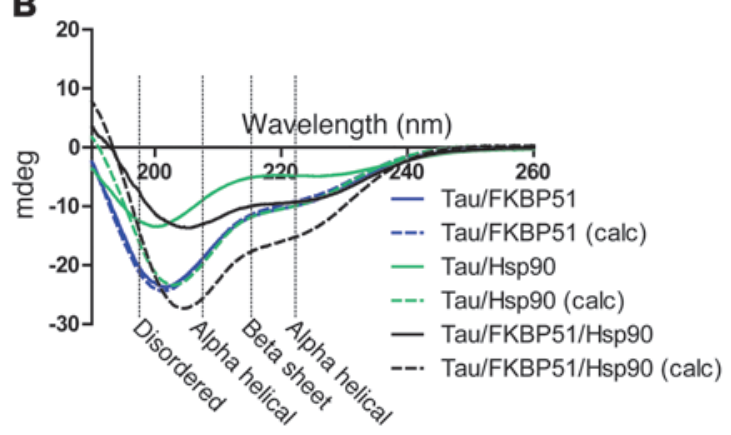

E

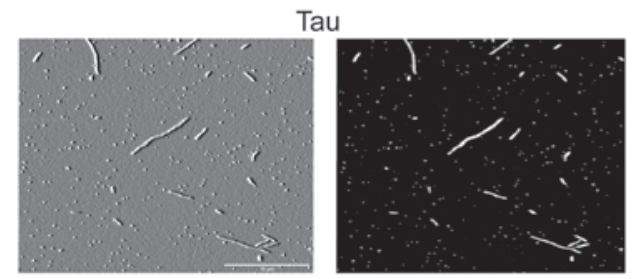

Tau/FKBP51

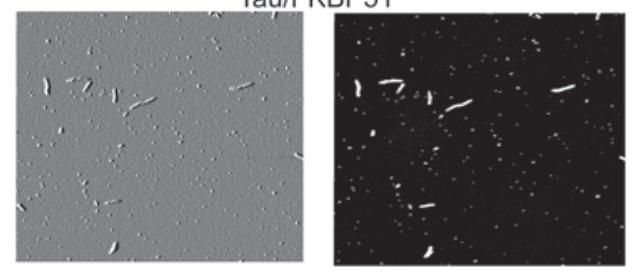

Tau/Hsp90

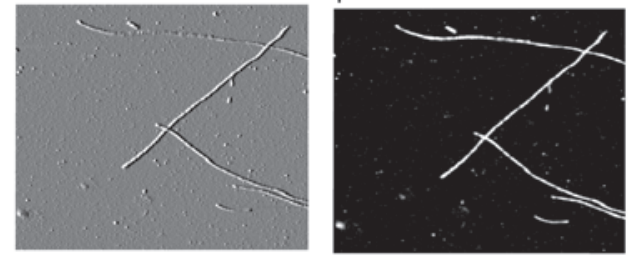

Tau/FKBP51/Hsp90
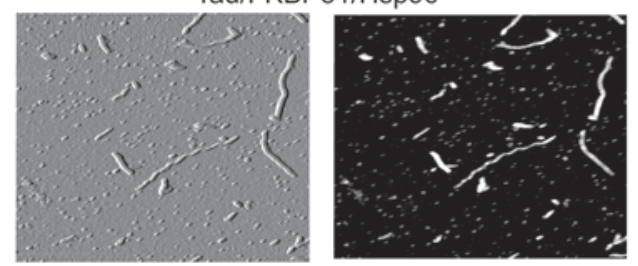

\section{Figure 3}

FKBP51 and Hsp90 combine to produce oligomeric tau structures. (A) CD spectra of tau, FKBP51, and Hsp90 recombinant protein. CD spectra were obtained at protein concentrations of $10 \mu \mathrm{M}$ tau, $2 \mu \mathrm{M}$ FKBP51, and $3 \mu \mathrm{M}$ Hsp90 in $10 \mathrm{mM}$ sodium phosphate buffer (pH 7.5) at room temperature. (B) CD spectra readout of tau incubated with FKBP51, Hsp90, or both (solid lines). Calculated (calc) CD spectra (dashed lines) from the addition of each protein's spectra shows what the readout would display if there were not protein interactions. Measurements of tau filament formation were monitored daily for 6 days, with a reading taken at least every 16 hours. (C) Tau fibril assembly was measured ( \pm SEM) using DLS in the presence of FKBP51, Hsp90, and both combined. DLS measurements of tau filament formation were monitored daily for 6 days, with a reading taken at least every 16 hours. (D) Tau was stained with Thioflavin T (ThT) to show $\beta$-pleated sheet aggregation after 6 days of incubation alone or with FKBP51, Hsp90, or both combined $\left( \pm\right.$ SEM). ${ }^{\star \star \star} P<0.0001$. (E) Atomic force microscopy images of tau multimers. The product of the DLS was imaged using atomic force microscopy for conformation. Both height (black) and amplitude (gray) images are shown. Scale bar: $0.5 \mu \mathrm{m}$. (F) Recombinant proteins were applied to a dot blot after 6 days, with heparin incubation at a ratio of 35 parts tau to 1 part chaperone combination. This was probed with $\mathrm{T} 22$ and $\mathrm{H} 150$ (total tau) antibodies.

to occur. These calculated spectral plots were obtained by summing the spectra from each of the individual measurements taken for tau, FKBP51, or Hsp90; however, the observed CD spectra (Figure 3B, shown as solid lines) were in contrast to the calculated spectra, suggesting that structural changes were occurring when these proteins were combined. While FKBP51 alone (3:10 ratio of FKBP51 to tau) had no effect on the secondary structure of tau (Figure 3B), Hsp90 (2:10 ratio of Hsp90 to tau) did alter tau structure. Interestingly, when FKBP51, tau, and Hsp90 were incubated together at a respective 3:10:2 ratio, tau showed a unique structural change unlike that 
A

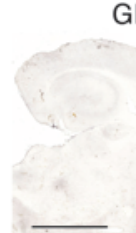

GFP
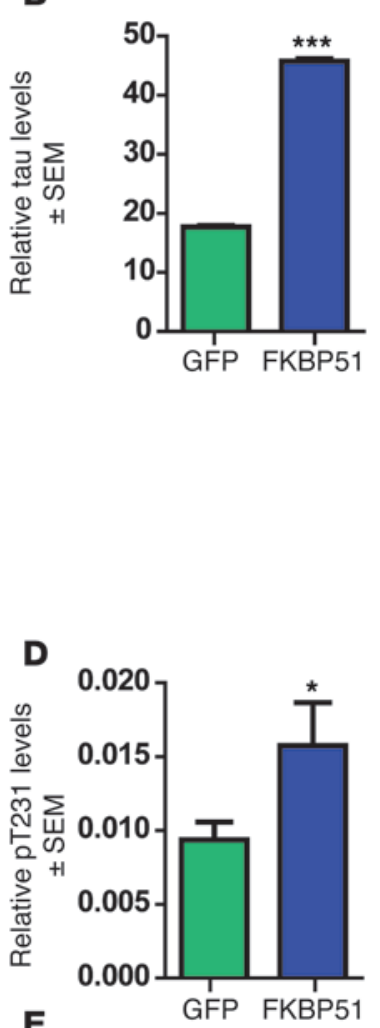

E

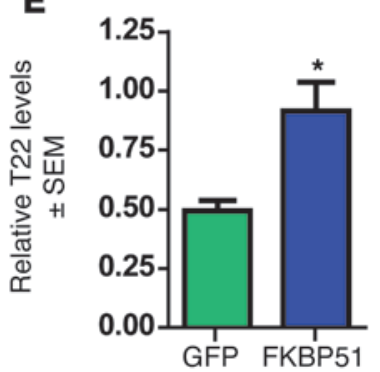

C

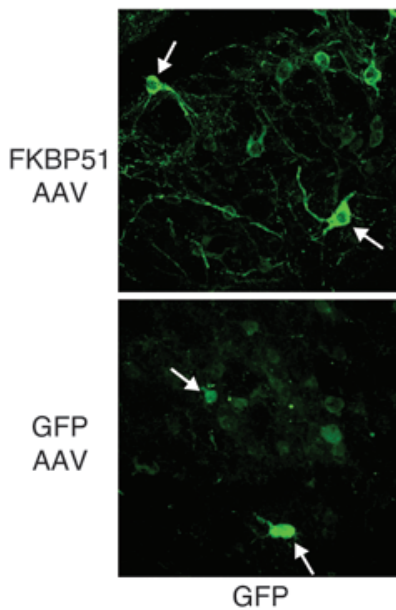

GFP
Tau

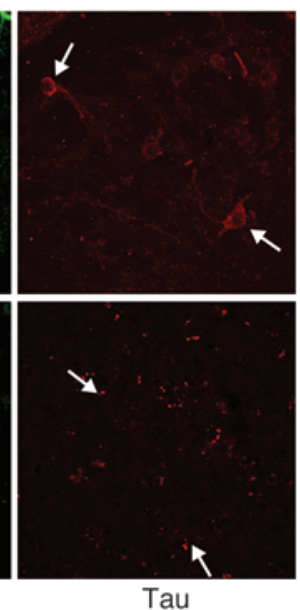

FKBP51
FKBP51
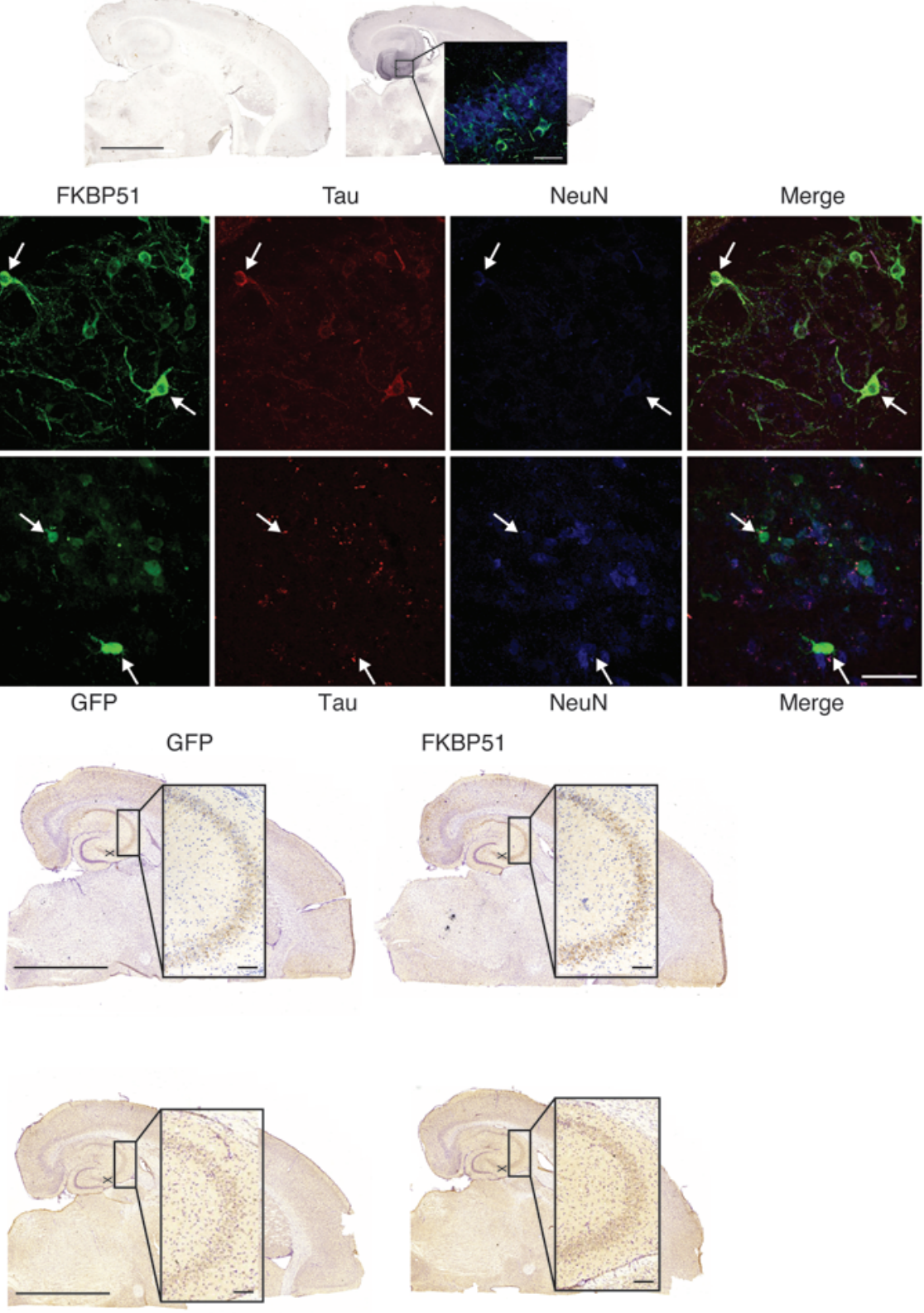

GFP AAV9 G
$\mathbf{F}$

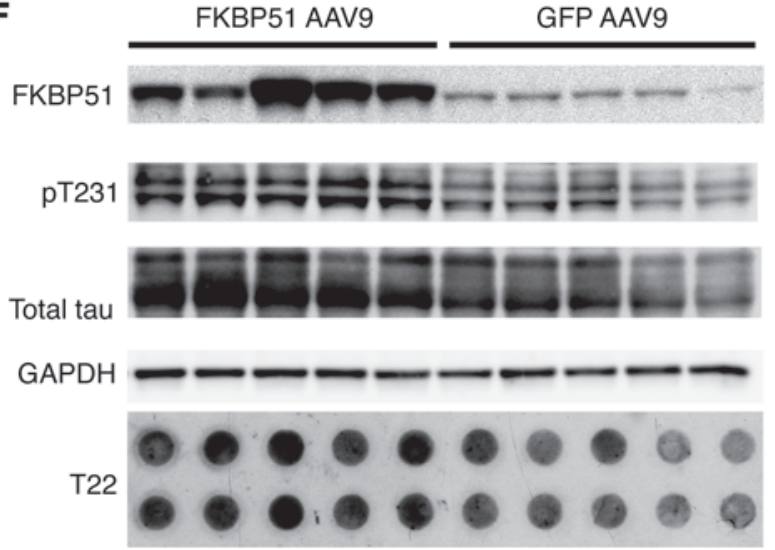

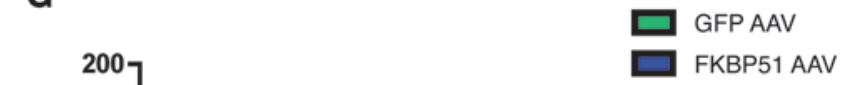

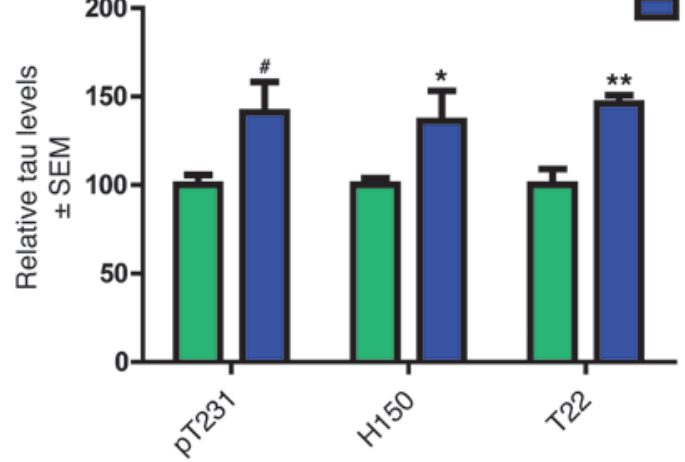




\section{Figure 4}

FKBP51 overexpression by viral vector in $\mathrm{rTg} 4510$ mice preserves tau. (A) GFP or FKBP51 was overexpressed in the HPC of rTg4510 mice using an AAV9 vector. Scale bar: 2,000 $\mu \mathrm{m} ; 50 \mu \mathrm{m}$ (inset). Original magnification, $\times 63$ (inset). (B) Total tau ( \pm SEM) was measured using ImageJ in neurons expressing FKBP51 or GFP. At least 15 images were taken per animal. ${ }^{* *} P<0.0001$. (C) Representative images FKBP51- and GFP-expressing neurons (arrows) (original magnification, $\times 63$ ) using antibodies toward GFP (green), FKBP51 (green), total Tau (red), and NeuN (blue). Scale bar: $50 \mu \mathrm{m}$. (D) Tissue from injected mice was stained for pT231 tau. pT231-positive area ( \pm SEM) was measured per CA3. ${ }^{\star} P=0.0463$. Representative sections are shown. Scale bar: 2,000 $\mu \mathrm{m} ; 200 \mu \mathrm{m}$ (inset). Original magnification, $\times 10$ (inset). (E) T22 oligomeric tau ( \pm SEM) was measured in the CA3 region of FKBP51- and GFP-overexpressing mice. ${ }^{*} P=0.0176$. Scale bar: $2,000 \mu \mathrm{m} ; 200 \mu \mathrm{m}$ (inset). Original magnification, $\times 10$ (inset). (F) Representative Western blot of brain homogenate from FKBP51- or GFP AAV-injected mice immunoblotted with FKBP51, pT231, H150 (total tau), and GAPDH. T22 antibody on complimentary dot blot. (G) Quantification of Western blots ( \pm SEM). ${ }^{*} P=0.03, \mathrm{pT} 231 ;{ }^{*} P=0.05$, H150 (total tau); ${ }^{\star \star} P=0.002$, T22.

after incubation with Hsp90 or FKBP51 alone, suggesting that FKBP51 only affected tau structure when Hsp90 was present. It is important to note that these spectra changes are still present when the calculated spectra are scaled to the global minima (Supplemental Figure 2A). Formal calculations of predicted secondary structure were performed using DichroWeb (39), and these are included in the supplement (Supplemental Figure 2B).

Since altering the structure of aggregation-prone proteins is known to contribute to their pathogenic assembly into fibrillar structures (40-43), we speculated that Hsp90 alone and Hsp90 with
FKBP51 could alter tau fibril assembly. We used dynamic light scattering (DLS) to assess how the Hsp90/FKBP51 complex was regulating the kinetics of global tau aggregation (44). For these studies, tau aggregation was induced with heparin sulfate in the presence of Hsp90 or FKBP51 alone and in the presence of Hsp90 and FKBP51 together. A 50:1 tau/chaperone ratio was used to minimize any contributions of the chaperones themselves to the signal produced. Tau combined with these Hsp90/FKBP51 mixtures was allowed to polymerize over a period of 6 days, and particle size distributions were determined with DLS throughout the duration of the experiment. Similar to the results using CD spectroscopy, FKBP51 alone did little to impact tau aggregation kinetics; both tau alone and tau with FKBP51 reached the half-maximal size by $2.3 \pm 0.3$ days (Figure $3 \mathrm{C}$ ). The rate of aggregation was also unaffected $\left(k_{\text {app }}=1.7 \pm 0.3\right.$ days $\left.^{-1}\right)$. Hsp90 alone actually accelerated tau aggregation (time for half-maximal size, $t_{\text {half }}=1.5 \pm 0.0$ days; apparent rate, $k_{\text {app }}=3.3 \pm 0.0$ days $^{-1}$ ) consistent with previous findings (20). Surprisingly, though, FKBP51 was able to slow the Hsp90-induced tau aggregation (time for halfmaximal size, thalf $=2.1 \pm 0.1$ days; apparent rate, $k_{\text {app }}=2.0 \pm 0.5$ days $^{-1}$ ) (Figure 3C), further supporting our hypothesis that FKBP51 uses Hsp90 to regulate tau biology and specifically promote its pathogenesis. Incubating these tau aggregates with Thioflavin $\mathrm{T}$, a dye that specifically recognizes $\beta$-pleated sheet amyloid structure, showed that Hsp90 promoted tau $\beta$-sheet aggregates, but FKBP51 prevented this (Figure 3D). Therefore, FKBP51 prevents tau from adopting $\beta$-sheet structure in the presence of Hsp90, promoting the production of amorphous tau aggregates. These results were confirmed using atomic force microscopy (Figure 3E). To determine whether recombinant tau was indeed forming oligomeric structures, tau was incubated with FKBP51 or Hsp90 alone or with FKBP51 and Hsp90 together with heparin, as described for the DLS exper-

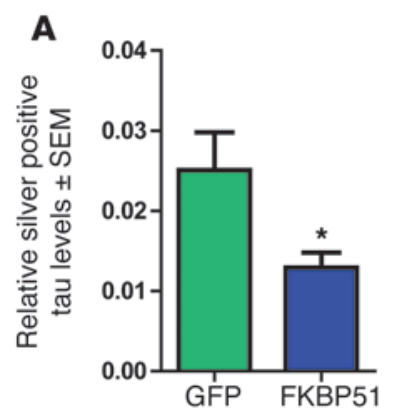

GFP
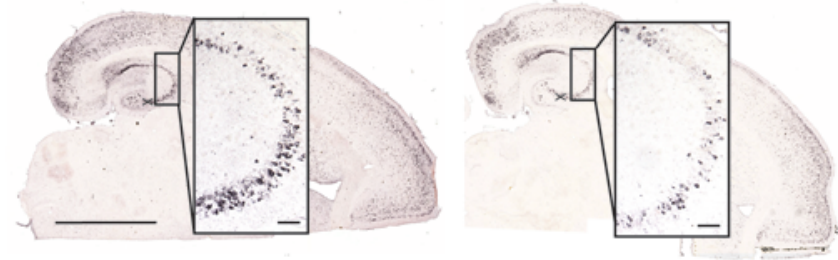

C

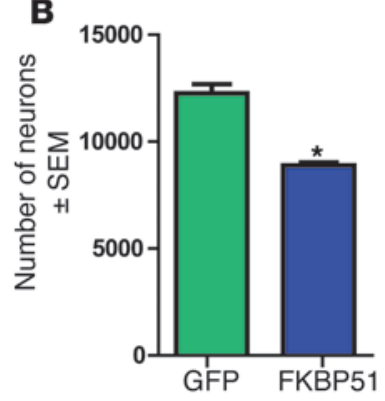

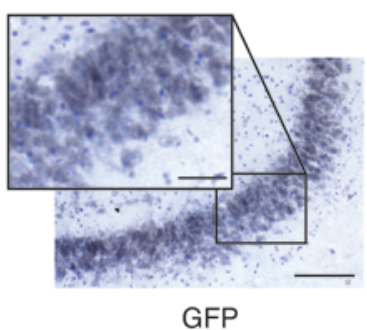

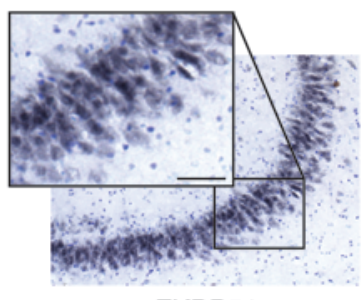

FKBP51

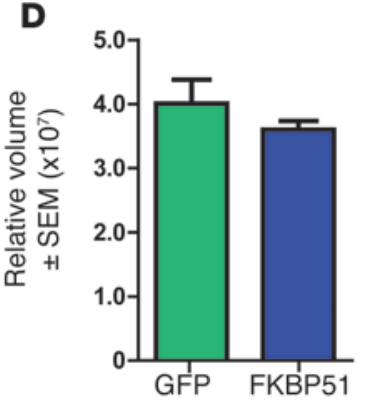

Figure 5

FKBP51 selectively promotes tau neurotoxicity. (A) Sections from FKBP51- and GFP AAV-injected mice were stained with Gallyas silver stain and positive area $\left( \pm\right.$ SEM) per CA3 was measured. ${ }^{*} P=0.0297$. Representative images of each group are shown. Scale bar: $2,000 \mu \mathrm{m} ; 200 \mu \mathrm{m}$ (inset). Original magnification, $\times 10$ (inset). (B) Neurons stained by cresyl violet and NeuN were counted $( \pm$ SEM) by stereology in the CA3 region. ${ }^{\star} P=0.0003$. (C) Representative images are shown. Scale bar: $500 \mu \mathrm{m} ; 50 \mu \mathrm{m}$. Original magnification, $\times 20$ (inset). (D) Volume of CA3 ( \pm SEM) in injected transgenic mice was evaluated by stereology. 


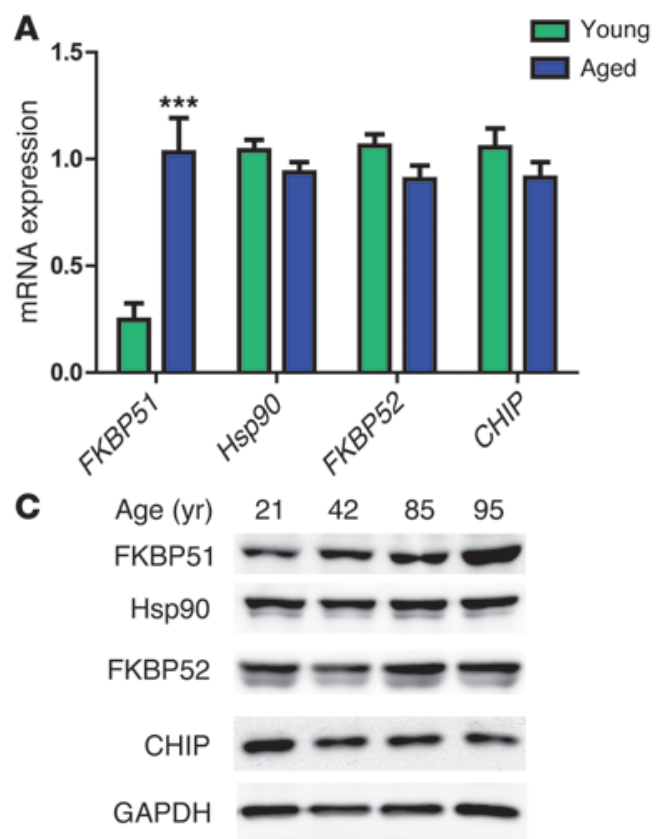

$\mathbf{E}$

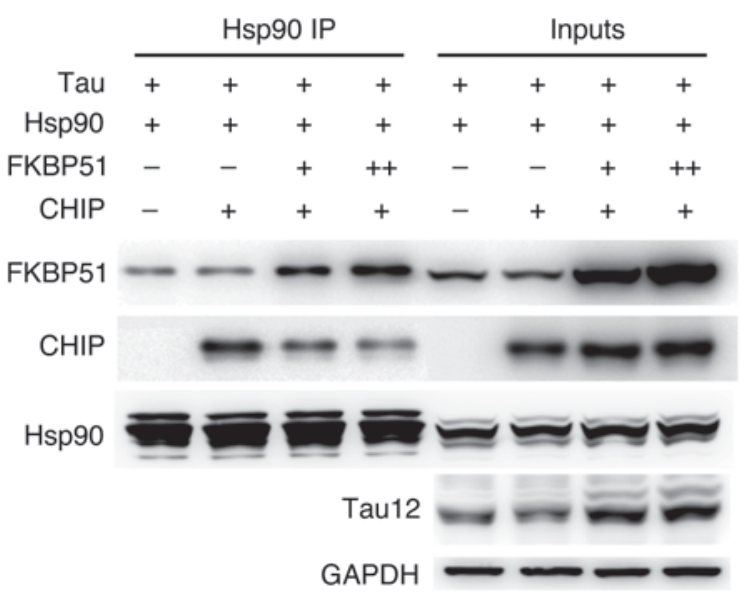

B
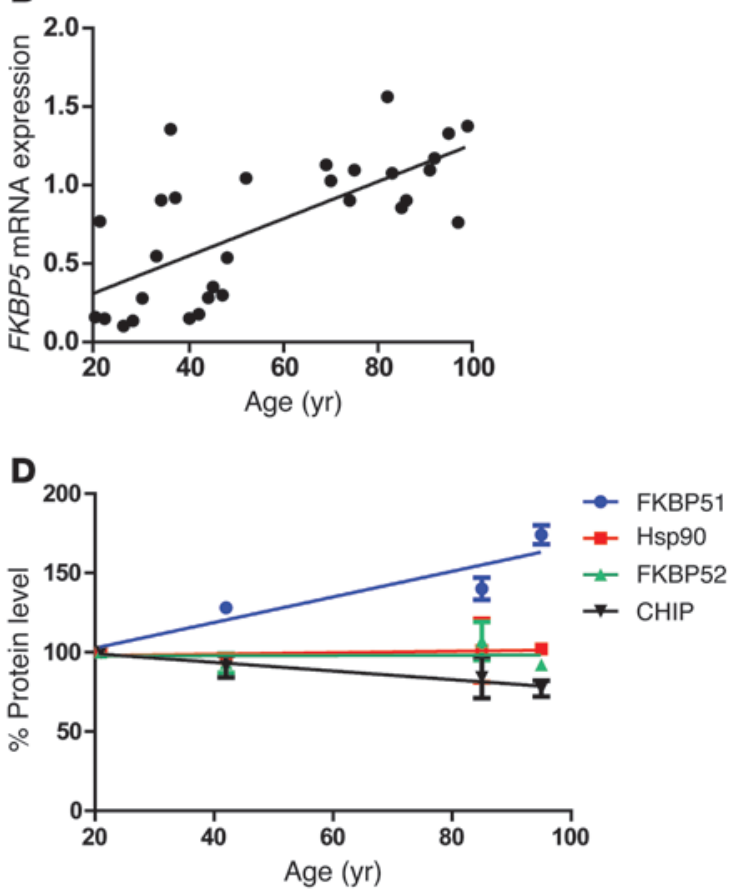

Figure 6

FKBP51 expression increases with age, altering Hsp90 complexes. (A) Average mRNA expression ( \pm SEM) from the SFG, PCG, EC, and HPC of young (20-59 years old) and aged (69-99 years old) samples. ${ }^{* *} P<0.0001$. (B) The average FKBP5 mRNA expression from all 4 regions for each sample was plotted against age. $P<0.0001 ; r=0.4916$. (C) Human brain homogenates from the PCG of 4 males (ages 21, 42, 85, and 95 years) were analyzed by Western blot by FKBP51, Hsp90, FKBP52, CHIP, and GAPDH antibodies. (D) Quantification of protein levels with aging from multiple blots. FKBP51, $P=0.0765, r=0.8528$; Hsp90, $P=0.394, r=0.3672 ;$ FKBP51, $P=0.9632, r=0.001352 ; \mathrm{CHIP}, P=0.0269, r=0.9469$. (E) Hek cells transfected with Tau, FKBP51, Hsp90, and CHIP, respectively, were harvested after 48 hours. Following mouse anti-Hsp90 antibody immunoprecipitation, samples were analyzed by Western blot for FKBP51, CHIP, Hsp90, Tau 12 (total tau), and GAPDH antibodies, respectively.

iment. The mixtures of these proteins were then analyzed by dot blot with the anti-tau oligomer-specific antibody, T22 (3). Indeed, T22-positive tau was only observed in the presence of FKBP51. The production of this oligomeric species was enhanced, even though total tau levels were the same, when FKBP51 and Hsp90 were incubated together with tau (Figure 3F). Hsp90 alone produced no T22 immunoreactivity. In this way, FKBP51 causes Hsp90 to alter its structural influence on tau, leading to the formation of nonamyloid tau aggregates and oligomeric tau species, which have both been implicated as the primary toxic tau species that facilitate neurodegeneration $(4,5,45,46)$. Based on these findings, we speculated that FKBP51 overexpression in the brain may drive tau toxicity. Therefore, we sought to evaluate the effects of FKBP51 in vivo.
In vivo, FKBP51 overexpression preserves tau species, including oligomers. The hippocampi (HPC) of a cohort of 4-month-old rTg4510 transgenic mice, which express human P301L mutant human tau, were injected bilaterally with AAV9 particles expressing either FKBP51 $(n=5)$ or GFP $(n=5)$. These mice have abundant Hsp90 levels and low basal FKBP51 levels at 6 months of age, and they are the best model to recapitulate human tau pathobiology (5, 45, 47-49). Therefore, 2 months following these injections, 6-month-old mice were harvested and examined histologically, as previously described (47). FKBP51 expression was observed in the HPC; however, the AAV did not transduce all neurons (Figure 4A). Due to this typical limitation of $\mathrm{AAV}$ use, a fluorescent masking technique previously developed by our lab was used to evaluate the levels of tau in neurons express- 
A

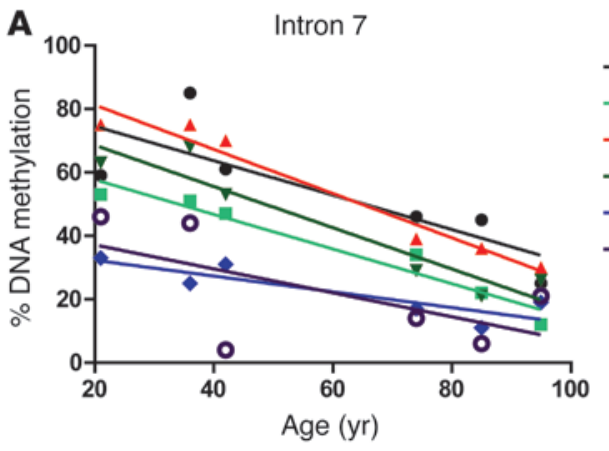

B
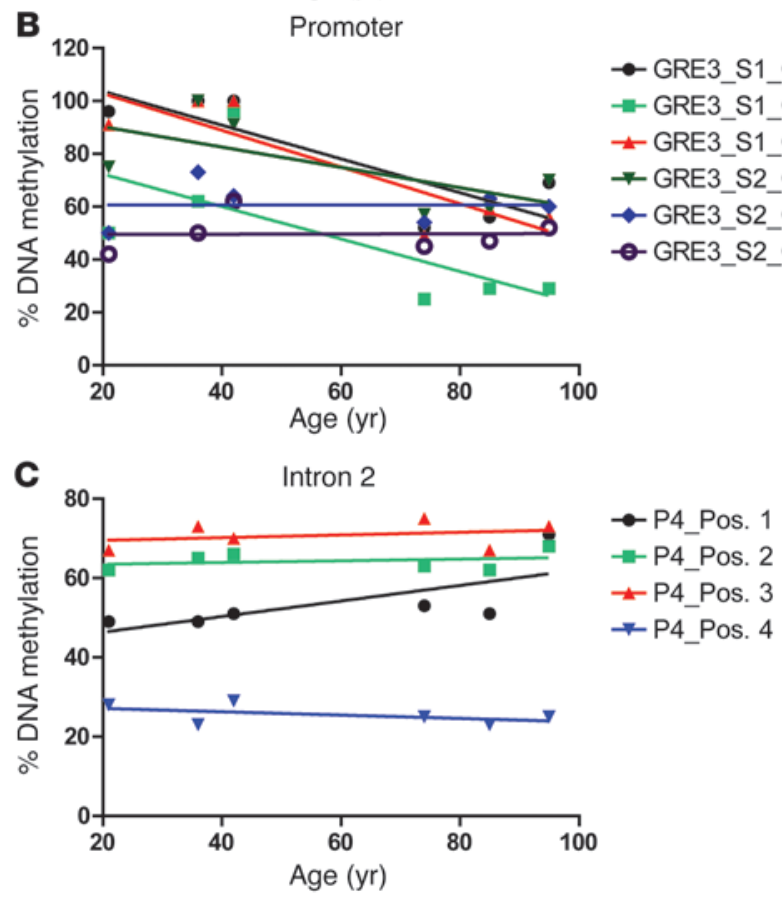

ing the AAV product, in this case FKBP51 or GFP (44). Triple-immunofluorescent staining of sections for FKBP51, total tau, and the neuronal marker, NeuN, revealed that the amount of tau found within FKBP51-positive neurons was significantly increased compared with tau levels in neurons overexpressing GFP (Figure 4, B and C). In fact upon further analysis of the site surrounding the injection site, the levels of phosphorylated tau throughout the CA3 region were significantly higher in mice injected with FKBP51 compared with those injected with GFP (Figure 4D). Based on our in vitro results, tissue was then stained with the oligomeric tau antibody, T22 (3). T22 tau was significantly increased in the CA3 region of FKBP51injected mice, compared with that in GFP-injected littermates (Figure 4E), indicating that FKBP51 was preserving tau in an oligomeric state. To further confirm the accumulation of tau by FKBP51 in vivo, homogenized tissue from FKBP51- or GFP AAV9-injected mice was analyzed by Western blot and dot blot (Figure 4F). Both phospho-tau (pT231) and total tau (H150) were significantly preserved in mice injected with FKBP51 AAV9 particles compared with GFP AAV9-injected littermates. In addition, T22 oligomeric tau was significantly preserved in mice overexpressing FKBP51 (Figure 4G).

FKBP51 prevents tangle formation but enhances tau neurotoxicity. Our in vitro data suggested that FKBP51 also prevented amyloid formation. The rTg4510 mouse is one of the only transgenic models

\section{Figure 7}

FKBP5 DNA demethylation with aging. Average FKBP5 methylation analysis of human DNA samples from the PCG of individuals aged $21,36,42,74,85,95$ years were performed on multiple CpG sites in (A) intron 7, (B) promoter, and (C) intron 2. Significant demethylation in intron 7 was found at P1_S1_Pos2 $(P=0.0012, r=0.6506), \mathrm{P} 1 \_S 2$ Pos1 $(P=0.0007, r=0.9585)$, P1_S2_Pos2 $(P=0.0039, r=0.9002)$, P1_S2_Pos3 $(P=0.0240, r=0.9002)$, and P1_S2_Pos3 $(P=0.0240$, $r=0.7583)$. Methylation was significantly decreased in the promoter at GRE_S1_CG1 $(P=0.0327, r=0.7199)$ and GRE3_S1_CG3 $(P=0.0216$, $r=0.7698)$.

to produce Gallyas silver-positive tangles that are a traditional pathological hallmark for AD and other tauopathies (47). Gallyas silver stains the same structure as Thioflavin T (50), but Gallyas lacks the fluorescent properties of Thioflavin $\mathrm{T}$. Thus, the previous characterization of Gallyas silver in this model combined with its lack of fluorescence in the GFP spectrum made it the ideal stain to assess $\beta$-sheet structure in these studies. Silver-positive tau was significantly reduced in the CA3 region of mice injected with FKBP51 AAV compared with that in those injected with GFP AAV (Figure 5A). Thus, FKBP51 overexpression in neurons promoted the accumulation of nonamyloid oligomeric tau species, similar to what was observed in vitro.

The toxicity of these T22-positive/silver-negative tau species produced by FKBP51 overexpression were then directly evaluated using unbiased stereology. Indeed, in $\mathrm{rTg} 4510$ mice expressing FKBP51, there was a significant decrease in the number of neurons in the CA3 hippocampal region compared with that in mice injected with GFP AAV (Figure 5, B and C). This neuron loss did not significantly alter CA3 volume (Figure $5 \mathrm{D}$ ). Since this was the first time that FKBP51 and tau have been shown to be neurotoxic, we performed in vitro experiments to confirm this result. Primary neurons from tau-expressing pups were transduced with FKBP51 AAV9 particles and allowed to express for 14 days. On the 14th day, an MTS assay was performed to measure toxicity. Significant toxicity was found in tau-expressing neurons transduced with FKBP51 AAV9 compared with GFP AAV9-transduced controls (Supplemental Figure 3). Additionally, Neuro2A cells, a mouse neuroblastoma line, were transfected with tau, FKBP51, or the combination for 48 hours. MTS assays performed at the end of the 48 hours revealed significant cell toxicity in the neuronal line, but only in the cells transfected with tau and FKBP51 together (Supplemental Figure 3).

Increasing FKBP51 levels with age allows a shift in Hsp90 functionality. We then sought to determine whether FKBP51 levels could increase with age or disease, perhaps linking FKBP51 to tau pathogenesis. FKBP51 mRNA expression was measured via microarray analyses (51) in young (ages 20 to 59 years; $n=22$, mean $35.4 \pm 10.5$ years) and aged (ages 69 to 99 years; $n=33$, mean $84.2 \pm 8.9$ years) nonneurologically diseased human brain tissue from the following regions: the postcentral gyrus (PCG), entorhinal cortex (EC), HPC, and superior-frontal gyrus (SFG). FKBP51 expression was significantly increased with age (Figure 6A). In fact, there was a 3- to 4-fold increase in FKBP51 expression in all 4 regions in the aged samples (Supplemental Figure 4A). Hsp90 expression levels were unchanged with aging (Figure 6A), with the exception of those in the SFG (Supplemental Figure 4B), which showed a significant decrease in expression. In contrast to FKBP51 expression, FKBP52 and CHIP, 2 other TPR Hsp90 cochaperones that could 
A

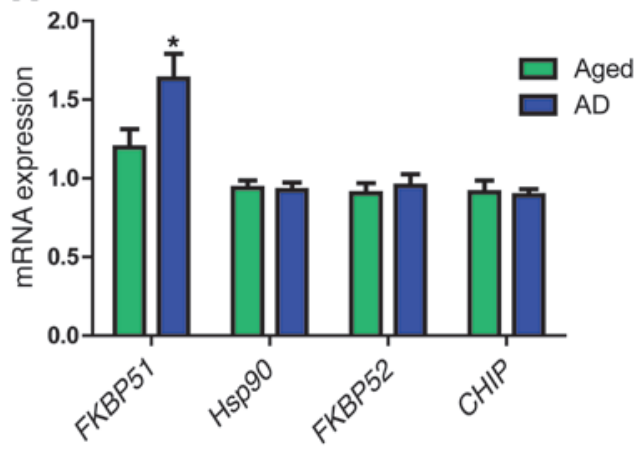

C

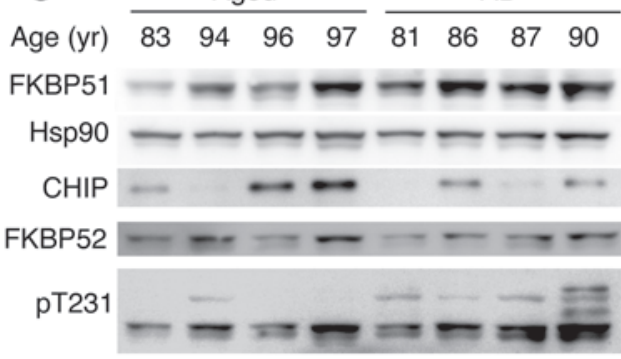

Total tau
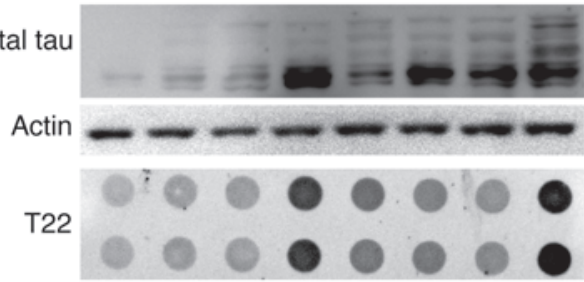

B

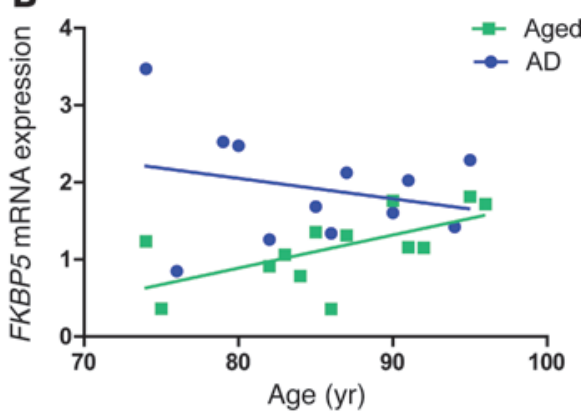

D

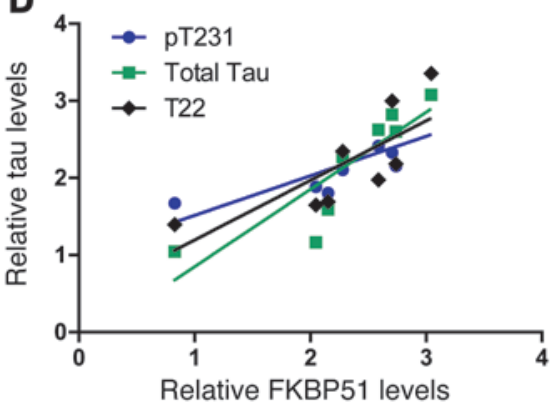

E

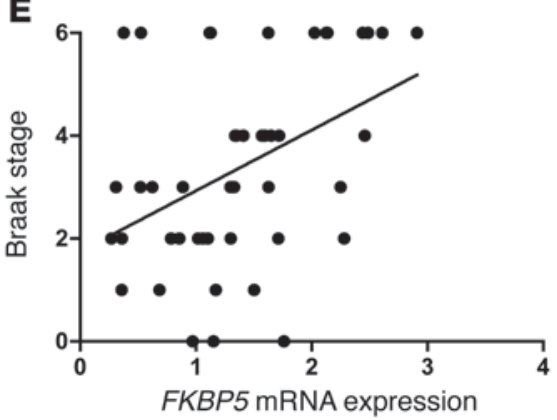

Figure 8

FKBP51 levels increase in AD samples, leading to chaperone imbalance. (A) Average mRNA expression ( \pm SEM) from the SFG, PCG, EC, and HPC of $A D$ and age-matched (69-99 years) samples. ${ }^{*} P=0.0306$. (B) FKBP5 expression correlation with aging in $A D$ and age-matched samples. (C) A representative image from Western blot analysis of the medial temporal gyri of 4 male aged and $A D$ samples. Blots were probed with FKBP51, Hsp90, CHIP, FKBP52, H150 (total tau), pT231, and actin antibodies. (D) Quantification of total levels, as they correlate to FKBP51 levels. $P=0.0203$, pT231; $P=0.0045$, $\mathrm{H} 150$ (total tau); $P=0.0251$, T22. (E) Linear regression of FKBP5 expression levels with Braak staging. The average mRNA expression from all 4 regions for each sample was plotted against Braak stage. $P=0.0036 ; r=0.1774$. compete with FKBP51 for binding to Hsp90 (29), had decreasing trends (Figure 6A), but were not significantly decreased with age in any region measured (Supplemental Figure 4, C and D). Additionally, we found that increasing FKBP51 expression levels were significantly correlated with age (Figure 6B). This was consistent with FKBP51 expression data observed in human prefrontal cortex through the BrainCloud program (13). Similar results were observed at the protein level in the PCG by Western blot (Figure 6, C and D). Thus, the aging brain promotes an environment in which FKBP51 can dominate the Hsp90 machine, potentially leading to tau pathogenesis.

Based on these data, we hypothesized that in human tauopathies, increasing levels of FKBP51 could allow it to usurp Hsp90 from other cochaperones with lower affinity, causing it to produce toxic tau oligomers, rather than sequester tau into more benign amyloid fibrils (29). Indeed, in cells expressing constant levels of CHIP, an Hsp90 cochaperone that can facilitate tau clearance through the proteasome $(52,53)$, increasing FKBP51 levels were able to displace CHIP from Hsp90 and preserve tau levels (Figure 6E). When taken together, these data suggest that elevated FKBP51 can overtake the Hsp90 machinery in the aging brain and alter tau structure in a way that facilitates its neurotoxic accumulation.
FKBP5 DNA methylation decreases with aging, a mechanism for its increased expression. It was recently shown that decreases in FKBP5 DNA methylation increase the expression of FKBP51 (54). To see whether this was the mechanism leading to the significantly increased FKBP51 expression found in the aged brain, methylation analysis using pyrosequencing was performed on DNA from PCG brain tissue of aging nondemented samples $(n=6)$ on multiple $\mathrm{CPG}$ sites in functional glucocorticoid response elements in intron 7 , intron 2, and the promoter (Figure 7) of FKBP5 (54). While intron 2 showed no significant linear decrease in methylation with aging, intron 7 and the promoter had 7 CPG sites for which DNA methylation was significantly decreased with aging. Significant demethylation in intron 7 was found at $5 \mathrm{CpG}$ positions (Figure 7A). Methylation was significantly decreased in the promoter in 2 locations (Figure 7B). These age-associated decreases in DNA methylation in specific regulatory elements could explain why FKBP51 expression is progressively increased with age (Figure 6E).

FKBP51 expression is further increased in the AD brain, perhaps through altered FKBP5 DNA methylation. Given the role of FKBP51 on tau accumulation and its pathogenicity, we examined whether FKBP51 levels were elevated further in $\mathrm{AD}$ brain compared with those in normal age-matched controls. FKBP51 mRNA expression was mea- 
A

A
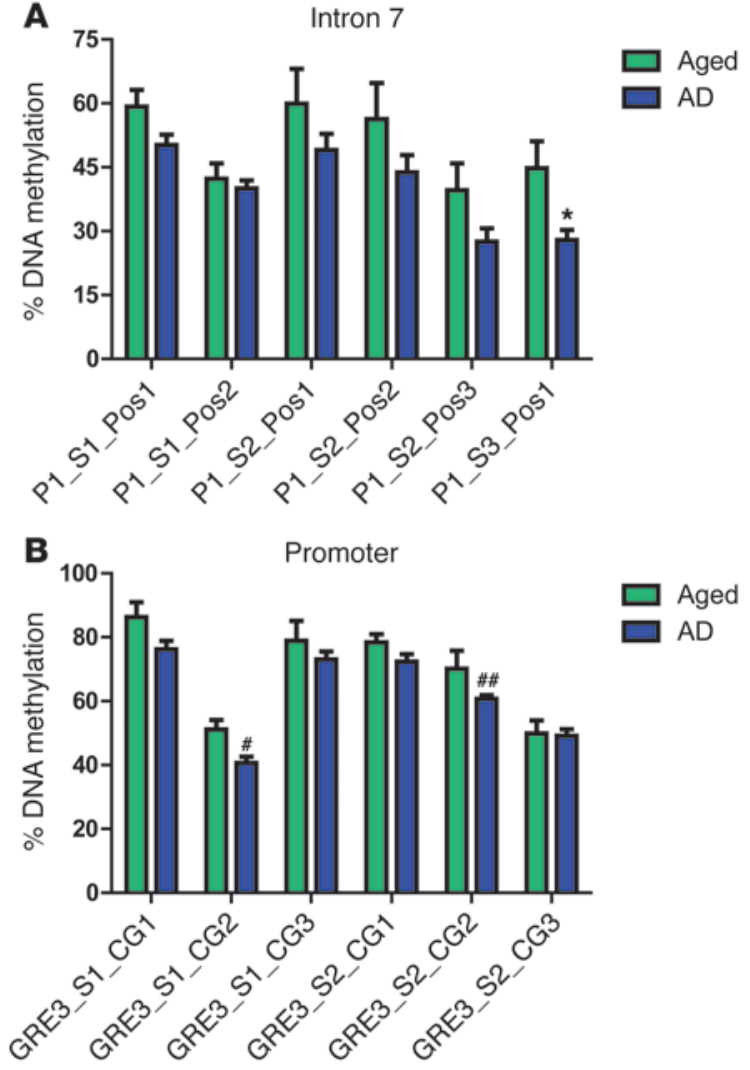

C ${ }_{80}$ Intron 2

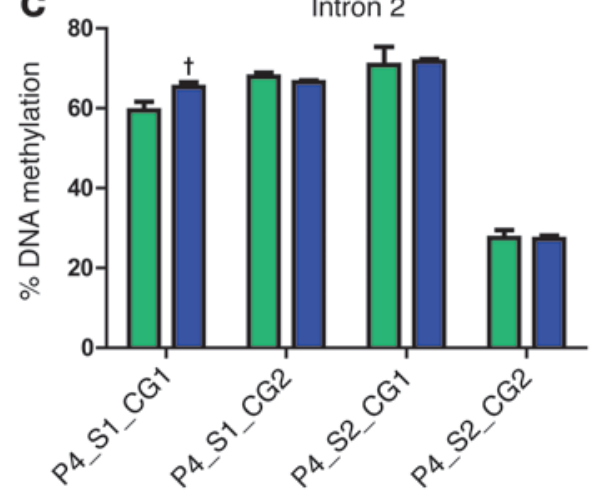

sured using microarray analyses of the PCG, EC, HPC, and SFG of brain samples individually diagnosed with $\mathrm{AD}(n=26,74-95$ years old; mean age $85.7 \pm 6.5$ years) and age-matched control samples $(n=33$, age $69-99$ years, average age $84.2 \pm 6.5$ years $)(51)$. FKBP51 expression was significantly increased in samples of diagnosed $A D$ compared with that in age-matched controls, while the levels of Hsp90 and other TPR-containing proteins were unchanged with disease state (Figure 8A). FKBP51 expression was much higher in younger individuals with $\mathrm{AD}$ (aged $70-80$ years), but levels of FKBP51 in older individuals with $\mathrm{AD}$ (>80 years) were indistinguishable from older nondemented individuals (Figure 8B). This suggests that AD accelerates age-associated FKBP51 upregulation. Protein levels were also evaluated in a subset of these cases using medial temporal gyrus tissue. FKBP51 expression was increased in the samples of diagnosed $\mathrm{AD}$, compared with that in the age-matched cognitively normal samples (Figure 8, C and D).

\section{Figure 9}

FKBP5 methylation is decreased in $A D$ brain. Average methylation ( \pm SEM) analysis of human DNA from the medial temporal gyrus of $A D$ and agematched samples from multiple CpG sites from (A) intron 7, (B) the promoter, and (C) intron 2 of $F K B P 5$. Significant demethylation in AD genotype was found in intron 7 at P1_S3 Pos $1\left({ }^{*} P=0.0243\right)$ and in the promoter at GRE_S1_CG2 (\#P=0.0105) and GRE3_S2_CG3 (\# $P=0.0424)$. Significant methylation was found in intron 2 at $P 4 \_S 1 \_C G 1\left({ }^{\dagger} P=0.0244\right)$.

Hsp90 levels appeared unchanged, while the levels of FKBP52 and $\mathrm{CHIP}$ trended toward a decrease in $\mathrm{AD}$ samples. In addition, those samples with higher tau levels, regardless of disease diagnosis, also had overall higher FKBP51 levels, further supporting a link between FKBP51 and tau accumulation.

Based on these results, we speculated that increasing FKBP51 levels might correlate with disease progression or disease onset. To assess this possibility, correlation analyses were performed based on Braak staging. We chose Braak staging as the most unbiased measure for disease progression. Indeed, increased FKBP51 levels were significantly correlated with increased Braak staging, suggesting that FKBP51 may have a role in $\mathrm{AD}$ progression (Figure $8 \mathrm{E}$ ). Furthermore, when the aged samples were evaluated alone, the significant increase in Braak staging with FKBP51 expression was maintained (Supplemental Figure 5). In this way, FKBP51 may play a role in accelerating disease onset and progression by facilitating the production and accumulation of toxic tau, leading to $\mathrm{AD}$ and expediting its pathogenesis.

To evaluate the role of $\mathrm{AD}$ on FKBP5 DNA methylation, the same pyrosequencing analyses as described above were performed on brain tissue from the medial temporal gyrus of $\mathrm{AD}$ samples $(n=10)$ and those of aged-matched controls $(n=5)$. There was a significant decrease in methylation at $3 \mathrm{CpG}$ sites; 1 in intron 7 (Figure 9A) and 2 in the promoter (Figure 9B). There was only one position in which DNA methylation was increased in the $\mathrm{AD}$ population (Figure 9C). There was also a decreasing trend of overall FKBP5 methylation in intron 7 as well as the promoter areas at every tested $\mathrm{CPG}$ of $\mathrm{AD}$ diagnosed samples compared with nondemented age-matched controls (Supplemental Figure 6).

\section{Discussion}

We have found that a chaperone, FKBP51, can work with Hsp90 to produce oligomeric tau in the brain, facilitating its neurotoxicity. FKBP51 overexpression enhanced neuronal loss in the rTg4510 tau transgenic mouse model, suggesting that FKBP51 coordinates with Hsp90 to prevent tau clearance and facilitate its toxicity. This provides further evidence that other forms of tau, besides those that are pathologically visible in postmortem tissue like tangles, are highly neurotoxic $(5,45,55,56)$. Thus, factors that facilitate FKBP51 expression could promote an environment in the aging brain that supports tau pathogenesis. We show that FKBP5 methylation is reduced both with age and in disease, which could be one factor leading to increased FKBP51 expression $(54,57,58)$. FKBP5 methylation and FKBP51 expression appear to be inversely proportional over time. Therefore, it is certainly conceivable that age could combine with other factors, such as stress or genetic variation, to increase FKBP51 expression to detrimentally high levels. We found that FKBP51 levels were further enhanced in the $\mathrm{AD}$ brain compared with those in agematched controls, possibly resulting from FKBP5 demethylation in these functional regulatory elements, as shown previously (54). In fact, the progressive increases in FKBP51 levels correlated with Braak 
staging in the entire sample population as well as the nondemented aging subpopulation alone. Additionally, since the FKBP5 gene is responsive to stress hormone (54), the levels of which increase in $\mathrm{AD}$ (59), FKBP51 could be exploited as a novel biomarker to compliment other such molecules for evaluating AD risk.

Based on these findings, we predict that people predisposed to increased levels of FKBP51 could display a higher risk for AD onset and possibly a more rapid progression of the disease. On the other hand, it is possible that the pathogenic processes that manifest in AD facilitate increased FKBP51 expression as well, which could also explain why FKBP51 levels are further increased in individuals with $\mathrm{AD}$ compared with those in age-matched controls. Either scenario would likely create a vicious cycle in the brain that contributes to disease progression.

At the molecular level, these studies show that FKBP51 can prevent tau clearance through the $20 \mathrm{~S}$ proteasome without directly impairing 20S proteasomal activity. This is one of the few examples in which chaperones can coordinately interact with a substrate, in this case tau, to block its degradation. This type of control of tau degradation may be unique to tau because of its high level of disorder (60). Since the $20 \mathrm{~S}$ proteasome typically proteolyzes unfolded proteins (35), it is possible that FKBP51 and Hsp90 bind tau, giving it more order and preventing its indiscriminate degradation through the $20 \mathrm{~S}$ proteasome. But this appears to come at a price: FKBP51 and Hsp90 also promote a structure for tau that produces toxicity, supporting the idea of the need for balance in the proteostasis network for healthy neuronal aging and function (61). When chaperone levels are too high or too low, some substrates that are particularly difficult to handle can begin to accumulate and cause disease. These substrates can then have detrimental consequences to the proteostasis system and general cellular health (62-65).

Perhaps the most intriguing result from these studies is the discovery that a chaperone can mediate the formation of tau oligomers. These data also show that subverting amyloid formation with chaperones could produce a deleterious consequence. Recent work has begun to question the role of amyloids in $\mathrm{AD}$ (66). While amyloid pathology (tangles, plaques, Lewy bodies, etc.) is the hallmark of many neurodegenerative diseases, these types of pathologies do not correlate well with neuronal loss or dysfunction in animal models of disease. For example, in the rTg4510 tau transgenic mice, neurons with tau tangle structures are longer lived $(4,67)$. This same model displays cognitive recovery when tau is suppressed, despite continued tangle formation $(5,46)$. Moreover, strategies that deplete soluble intermediate tau species without impacting tangle pathology have been very successful at improving cognitive impairment and neuronal loss in transgenic tau models $(5,46,68)$. The current evidence suggests that sequestration of mis-

folded or abnormally folded proteins into amyloid $\beta$-sheet structures may be more beneficial to neurons than allowing these intermediates to traverse the intracellular milieu (69-71). FKBP51 not only interferes with tau degradation and promotes tau oligomer formation, but FKBP51 also impairs tau's ability to be sequestered into less toxic tangles, facilitating tau toxicity. This is why strategies aimed at depleting FKBP51 or inhibiting its association with tau or Hsp90 may be highly effective for tau-based therapeutics.

Overall, this study shows that a significant increase in FKBP51 levels in the aged and $\mathrm{AD}$ brain could promote an environment that preserves toxic tau oligomers. We show that FKBP51 coordinates with the Hsp90 machinery to alter tau structure, block its degradation, and promote abnormal oligomer formation. This strongly implicates FKBP51 as a therapeutic target for AD and other tauopathies. These data ultimately shed light on the nature of tau accumulation and support a new body of research, suggesting that chaperone proteins impede rather than aid in the pathogenesis of these diseases.

\section{Methods}

A full description of all materials and methods is available in the Supplemental Methods. See complete unedited blots in the supplemental material. Data for the microarray have been deposited in the Gene Expression Omnibus (GEO) database, with accession number GSE11882.

Statistics. To compare 2 groups, a Student's $t$ test was used. Groups larger than 2 were evaluated using a 1-way ANOVA test. Linear regression was used to show the relationship between 2 variables. $P$ values below 0.05 were considered significant.

Study approval. All studies were carried out following the guidelines set by the University of South Florida's Institutional Animal Care and Use Committee in accordance with the Association for Assessment and Accreditation of Laboratory Animal Care International regulations.

\section{Acknowledgments}

This work was supported by NIH/NINDS R01 NS073899, American Federation for Aging Research, and Brightfocus Foundation. Support for the University of California, Irvine, tissue bank was provided by the NIH/NIA Alzheimer's Disease Research Center. We thank Kevin Nash for the gift of AAV9-EGFP and Peter Mouton for stereology assistance.

Received for publication January 30, 2013, and accepted in revised form June 27, 2013.

Address correspondence to: Chad Dickey, University of South Florida, 4001 E. Fletcher Ave., MDC 36, Tampa, Florida 33613, USA. Phone: 813.396.0639; Fax: 813.974.3081; E-mail: cdickey@ health.usf.edu.

\footnotetext{
1. Braak H, Braak E. Neuropathological stageing of Alzheimer-related changes. Acta Neuropathol (Berl). 1991;82(4):239-259.

2. Gomez-Isla T, et al. Neuronal loss correlates with but exceeds neurofibrillary tangles in Alzheimer's disease. Ann Neurol. 1997;41(1):17-24.

3. Lasagna-Reeves CA, et al. Identification of oligomers at early stages of tau aggregation in Alzheimer's disease. FASEB J. 2012;26(5):1946-1959.

4. de Calignon $A$, et al. Caspase activation precedes and leads to tangles. Nature. 2010;464(7292):1201-1204.

5. Santacruz K, et al. Tau suppression in a neurodegenerative mouse model improves memory function. Science. 2005;309(5733):476-481.

6. de Calignon A, et al. Propagation of tau pathology in a model of early Alzheimer's disease. Neuron.
}

2012;73(4):685-697.

7. Clavaguera F, et al. Transmission and spreading of tauopathy in transgenic mouse brain. Nat Cell Biol. 2009;11(7):909-913.

8. Liu L, et al. Trans-synaptic spread of tau pathology in vivo. PLoS One. 2012;7(2):e31302.

9. Hutton $\mathrm{M}$, et al. Association of missense and $5^{\prime}$-splicesite mutations in tau with the inherited dementia FTDP-17. Nature. 1998;393(6686):702-705.

10. Ros R, et al. A new mutation of the tau gene, $\mathrm{G} 303 \mathrm{~V}$, in early-onset familial progressive supranuclear palsy. Arch Neurol. 2005;62(9):1444-1450.

11. Dumanchin C, et al. Segregation of a missense mutation in the microtubule-associated protein tau gene with familial frontotemporal dementia and parkinsonism. Hum Mol Genet. 1998;
7(11):1825-1829.

12. Gresham D, Dunham MJ, Botstein D. Comparing whole genomes using DNA microarrays. Nat Rev Genet. 2008;9(4):291-302.

13. Colantuoni $\mathrm{C}$, et al. Temporal dynamics and genetic control of transcription in the human prefrontal cortex. Nature. 2011;478(7370):519-523.

14. Thompson AD, et al. Analysis of the tau-associated proteome reveals that exchange of Hsp70 for Hsp90 is involved in tau degradation. ACS Chem Biol. 2012;7(10):1677-1686.

15. Sherman MY, Goldberg AL. Cellular defenses against unfolded proteins: a cell biologist thinks about neurodegenerative diseases. Neuron. 2001; 29(1):15-32.

16. Dickey CA, et al. The high-affinity HSP90-CHIP 
complex recognizes and selectively degrades phosphorylated tau client proteins. J Clin Invest. 2007;117(3):648-658

17. Jinwal UK, et al. The Hsp90 cochaperone, FKBP51, increases Tau stability and polymerizes microtubules. J Neurosci. 2010;30(2):591-599.

18. Luo W, et al. Roles of heat-shock protein 90 in maintaining and facilitating the neurodegenerative phenotype in tauopathies. Proc Natl Acad Sci U S A. 2007; 104(22):9511-9516.

19. Dickey CA, et al. HSP induction mediates selective clearance of tau phosphorylated at proline-directed Ser/Thr sites but not KXGS (MARK) sites. FASEBJ. 2006;20(6):753-755.

20. Tortosa E, Santa-Maria I, Moreno F, Lim F, Perez M, Avila J. Binding of Hsp90 to tau promotes a conformational change and aggregation of tau protein. J Alzheimers Dis. 2009;17(2):319-325.

21. Liou YC, et al. Role of the prolyl isomerase Pin 1 in protecting against age-dependent neurodegeneration. Nature. 2003;424(6948):556-561.

22. Pastorino L, et al. The prolyl isomerase Pin 1 regulates amyloid precursor protein processing and amyloid-beta production. Nature. 2006; 440(7083):528-534.

23. Koberle M, et al. Yersinia enterocolitica YopT and Clostridium difficile toxin B induce expression of GILZ in epithelial cells. PLoS One. 2012; 7(7):e40730

24. Binder EB, et al. Polymorphisms in FKBP5 are associated with increased recurrence of depressive episodes and rapid response to antidepressant treatment. Nat Genet. 2004;36(12):1319-1325.

25. Smith DF, Baggenstoss BA, Marion TN, Rimerman RA. Two FKBP-related proteins are associated with progesterone receptor complexes. J Biol Chem. 1993; 268(24):18365-18371.

26. Radanyi C, Chambraud B, Baulieu EE. The ability of the immunophilin FKBP59-HBI to interact with the $90-\mathrm{kDa}$ heat shock protein is encoded by its tetratricopeptide repeat domain. Proc Natl Acad SciU S A. 1994;91(23):11197-11201.

27. Blatch GL, Lassle M. The tetratricopeptide repeat: a structural motif mediating protein-protein interactions. Bioessays. 1999;21(11):932-939.

28. Pirkl F, Buchner J. Functional analysis of the Hsp90-associated human peptidyl prolyl cis/trans isomerases FKBP51, FKBP52 and Cyp40. J Mol Biol. 2001;308(4):795-806

29. Schulke JP, et al. Differential impact of tetratricopeptide repeat proteins on the steroid hormone receptors. PLoS One. 2010;5(7):e11717.

30. O'Leary JC, et al. A new anti-depressive strategy for the elderly: ablation of FKBP5/FKBP51. PLoS One. 2011;6(9):e24840.

31. Tranguch $\mathrm{S}$, et al. Cochaperone immunophilin FKBP52 is critical to uterine receptivity for embryo implantation. Proc Natl Acad Sci U S A. 2005; 102(40):14326-14331.

32. David DC, Layfield R, Serpell L, Narain Y, Goedert M, Spillantini MG. Proteasomal degradation of tau protein. J Neurochem. 2002;83:176-185.

33. Grune T, et al. Tau protein degradation is catalyzed by the ATP/ubiquitin-independent $20 \mathrm{~S}$ proteasome under normal cell conditions. Arch Biochem Biophys. 2010;500(2):181-188.

34. Shringarpure R, Grune T, Davies KJ. Protein oxidation and $20 \mathrm{~S}$ proteasome-dependent proteolysis in mammalian cells. Cell Mol Life Sci. 2001; 58(10):1442-1450.

35. Coux O, Tanaka K, Goldberg AL. Structure and functions of the 20S and 26S proteasomes. Annu Rev Biochem. 1996;65:801-847.

36. Schweers O, Schonbrunn-Hanebeck E, Marx A, Mandelkow E. Structural studies of tau protein and Alzheimer paired helical filaments show no evidence for beta-structure. J Biol Chem. 1994; 269(39):24290-24297.

37. Prodromou C, et al. Regulation of Hsp90 ATPase activity by tetratricopeptide repeat (TPR)-domain co-chaperones. EMBOJ. 1999;18(3):754-762.

38. Xue B, Dunbrack RL, Williams RW, Dunker AK, Uversky VN. PONDR-FIT: a meta-predictor of intrinsically disordered amino acids. Biochim Biophys Acta. 2010;1804(4):996-1010.

39. Whitmore L, Wallace BA. Protein secondary structure analyses from circular dichroism spectroscopy: methods and reference databases. Biopolymers. 2008;89(5):392-400.

40. Uversky VN, Li J, Fink AL. Evidence for a partially folded intermediate in alpha-synuclein fibril formation. J Biol Chem. 2001;276(14):10737-10744.

41. Munishkina LA, Fink AL, Uversky VN. Conformational prerequisites for formation of amyloid fibrils from histones. J Mol Biol. 2004;342(4):1305-1324.

42. Uversky VN, Fink AL. Conformational constraints for amyloid fibrillation: the importance of being unfolded. Biochim Biophys Acta. 2004;1698(2):131-153.

43. Chirita CN, Congdon EE, Yin H, Kuret J. Triggers of full-length tau aggregation: a role for partially folded intermediates. Biochemistry. 2005; 44(15):5862-5872.

44. Abisambra JF, et al. Phosphorylation dynamics regulate Hsp27-mediated rescue of neuronal plasticity deficits in tau transgenic mice. J Neurosci. 2010; 30(46):15374-15382.

45. Berger $Z$, et al. Accumulation of pathological tau species and memory loss in a conditional model of tauopathy. J Neurosci. 2007;27(14):3650-3662.

46. O'Leary JC, et al. Phenothiazine-mediated rescue of cognition in tau transgenic mice requires neuroprotection and reduced soluble tau burden. Mol Neurodegener. 2010;5:45

47. Dickey C, et al. Aging analysis reveals slowed tau turnover and enhanced stress response in a mouse model of tauopathy. Am J Pathol. 2009;174(1):228-238.

48. Ramsden M, et al. Age-dependent neurofibrillary tangle formation, neuron loss, and memory impairment in a mouse model of human tauopathy (P301L). J Neurosci. 2005;25(46):10637-10647.

49. Spires TL, et al. Region-specific dissociation of neuronal loss and neurofibrillary pathology in a mouse model of tauopathy. Am J Pathol. 2006; 168(5):1598-1607.

50. Sun A, Nguyen XV, Bing G. Comparative analysis of an improved thioflavin-s stain, Gallyas silver stain, and immunohistochemistry for neurofibrillary tangle demonstration on the same sections. J Histochem Cytochem. 2002;50(4):463-472.

51. Cribbs DH, et al. Extensive innate immune gene activation accompanies brain aging, increasing vulnerability to cognitive decline and neurodegeneration: a microarray study. J Neuroinflammation. 2012; 9:179.

52. Dickey CA, et al. Deletion of the ubiquitin ligase CHIP leads to the accumulation, but not the aggregation, of both endogenous phospho- and caspase-3-cleaved tau species. J Neurosci. 2006; 26(26):6985-6996.

53. Petrucelli L, et al. CHIP and Hsp70 regulate tau ubiquitination, degradation and aggregation. Hum Mol Genet. 2004;13(7):703-714.

54. Klengel $\mathrm{T}$, et al. Allele-specific FKBP5 DNA demethylation mediates gene-childhood trauma interactions. Nat Neurosci. 2013;16(1):33-41.

55. Spires-Jones TL, Kopeikina KJ, Koffie RM, de Calignon A, Hyman BT. Are tangles as toxic as they look? J Mol Neurosci. 2011;45(3):438-444.

56. Wittmann CW, et al. Tauopathy in Drosophila: neurodegeneration without neurofibrillary tangles. Science. 2001;293(5530):711-714

57. Lee RS, et al. Chronic corticosterone exposure increases expression and decreases deoxyribonucleic acid methylation of Fkbp5 in mice. Endocrinology. 2010;151(9):4332-4343.

58. Yang X, et al. Glucocorticoid-induced loss of DNA methylation in non-neuronal cells and potential involvement of DNMT1 in epigenetic regulation of Fkbp5. Biochem Biophys Res Commun. 2012; 420(3):570-575.

59. Davis KL, et al. Cortisol and Alzheimer's disease, I: Basal studies. Am J Psychiatry. 1986;143(3):300-305.

60. Lichtenberg B, Mandelkow EM, Hagestedt T, Mandelkow E. Structure and elasticity of microtubule-associated protein tau. Nature. 1988; 334(6180):359-362.

61. Balch WE, Morimoto RI, Dillin A, Kelly JW. Adapting proteostasis for disease intervention. Science. 2008; 319(5865):916-919.

62. Bennett EJ, Bence NF, Jayakumar R, Kopito RR. Global impairment of the ubiquitin-proteasome system by nuclear or cytoplasmic protein aggregates precedes inclusion body formation. Mol Cell. 2005; 17(3):351-365.

63. Bence NF, Sampat RM, Kopito RR. Impairment of the ubiquitin-proteasome system by protein aggregation. Science. 2001;292(5521):1552-1555.

64. Gillardon F, et al. The $20 \mathrm{~S}$ proteasome isolated from Alzheimer's disease brain shows post-translational modifications but unchanged proteolytic activity. J Neurochem. 2007;101(6):1483-1490.

65. Gregori L, Hainfeld JF, Simon MN, Goldgaber D. Binding of amyloid beta protein to the $20 \mathrm{~S}$ proteasome. J Biol Chem. 1997;272(1):58-62.

66. Eisenberg D, Jucker M. The amyloid state of proteins in human diseases. Cell. 2012;148(6):1188-1203.

67. Spires-Jones TL, et al. In vivo imaging reveals dissociation between caspase activation and acute neuronal death in tangle-bearing neurons. J Neurosci. 2008;28(4):862-867.

68. Oddo S, Billings L, Kesslak JP, Cribbs DH, LaFerla FM. Abeta immunotherapy leads to clearance of early, but not late, hyperphosphorylated tau aggregates via the proteasome. Neuron. 2004;43(3):321-332.

69. O'Leary JC, Koren J, Dickey CA. Neuronal life span versus health span: principles of natural selection at work in the degenerating brain. JMol Neurosci. 2011; 45(3):467-472.

70. Brunden KR, Trojanowski JQ, Lee VM. Evidence that non-fibrillar tau causes pathology linked to neurodegeneration and behavioral impairments. J Alzheimers Dis. 2008;14(4):393-399.

71. Marx J. Alzheimer's disease. A new take on tau. Science. 2007;316(5830):1416-1417. 